\title{
Nonrepetitive Colourings of Planar Graphs with $O(\log n)$ Colours
}

\author{
Vida Dujmović $^{\dagger}$ Fabrizio Frati ${ }^{\ddagger}$ Gwenaël Joret ${ }^{\S}$ David R. Wood ${ }^{\uparrow}$
}

Submitted: Feb 17, 2012; Accepted: Feb 19, 2013; Published: Mar 1, 2013

Mathematics Subject Classifications: 05C15; 05C10

\begin{abstract}
A vertex colouring of a graph is nonrepetitive if there is no path for which the first half of the path is assigned the same sequence of colours as the second half. The nonrepetitive chromatic number of a graph $G$ is the minimum integer $k$ such that $G$ has a nonrepetitive $k$-colouring. Whether planar graphs have bounded nonrepetitive chromatic number is one of the most important open problems in the field. Despite this, the best known upper bound is $O(\sqrt{n})$ for $n$-vertex planar graphs. We prove a $O(\log n)$ upper bound.
\end{abstract}

\section{Introduction}

A vertex colouring of a graph is nonrepetitive if there is no path for which the first half of the path is assigned the same sequence of colours as the second half. More precisely, a $k$-colouring of a graph $G$ is a function $\psi$ that assigns one of $k$ colours to each vertex of $G$. A path $\left(v_{1}, v_{2}, \ldots, v_{2 t}\right)$ of even order in $G$ is repetitively coloured by $\psi$ if $\psi\left(v_{i}\right)=\psi\left(v_{t+i}\right)$ for all $i \in[1, t]:=\{1,2, \ldots, t\}$. A colouring $\psi$ of $G$ is nonrepetitive if no path of $G$ is repetitively coloured by $\psi$. Observe that a nonrepetitive colouring is proper, in the sense that adjacent vertices are coloured differently. The nonrepetitive chromatic number $\pi(G)$ is the minimum integer $k$ such that $G$ admits a nonrepetitive $k$-colouring.

The seminal result in this field is by Thue [19], who in 1906 proved that every path is nonrepetitively 3-colourable. Nonrepetitive colourings have recently been widely studied;

\footnotetext{
${ }^{\dagger}$ School of Mathematics and Statistics \& Department of Systems and Computer Engineering, Carleton University, Ottawa, Canada (vdujmovic@math.carleton.ca). Supported by NSERC and an Endeavour Fellowship from the Australian Government.

${ }^{\ddagger}$ School of Information Technologies, The University of Sydney, Sydney, Australia (brillo@it.usyd.edu.au).

${ }^{\S}$ Département d'Informatique, Université Libre de Bruxelles, Brussels, Belgium (gjoret@ulb.ac.be). Supported by an Endeavour Fellowship from the Australian Government.

ISchool of Mathematical Sciences, Monash University, Melbourne, Australia (david.wood@monash.edu). Research supported by the Australian Research Council.
} 
see the surveys $[6,10,11]$. A number of graph classes are known to have bounded nonrepetitive chromatic number. In particular, trees are nonrepetitively 4-colourable [5, 14], outerplanar graphs are nonrepetitively 12-colourable [4, 14], and more generally, every graph with treewidth $k$ is nonrepetitively $4^{k}$-colourable [14]. Graphs with maximum degree $\Delta$ are nonrepetitively $O\left(\Delta^{2}\right)$-colourable $[3,8,10,13]$.

Perhaps the most important open problem in the field of nonrepetitive colourings is whether planar graphs have bounded nonrepetitive chromatic number. This question, first asked by Alon et al. [3], has since been mentioned by numerous authors [2, 4, 7, 9$14,16,18]$. It is widely known that $\pi(G) \in O(\sqrt{n})$ for $n$-vertex planar graphs ${ }^{1}$, and this is the best known upper bound. The best known lower bound is 11, due to Pascal Ochem; see Appendix A. Here we prove a logarithmic upper bound.

Theorem 1. For every planar graph $G$ with $n$ vertices,

$$
\pi(G) \leqslant 8\left(1+\log _{3 / 2} n\right)
$$

We now explain that the above open problem is solved when restricted to paths of bounded length. For $p \geqslant 1$, a vertex colouring of a graph $G$ is $p$-centered if for every connected subgraph $X$ of $G$, some colour appears appears exactly once in $X$, or at least $p$ colours appear in $X$. In a repetitively coloured path of at most $2 p-2$ vertices, there are at most $p-1$ colours each appearing at least twice. Thus the colouring is not $p$-centered. Equivalently, every $p$-centered colouring is nonrepetitive on paths with at most $2 p-2$ vertices. Nešetřil and Ossona de Mendez [17] proved that for every graph $H$ and integer $p \geqslant 1$, there exists an integer $c$, such that every graph with no $H$-minor has a $p$-centered colouring with $c$ colours. This shows that (with $H=K_{5}$ ) for every integer $p \geqslant 1$, there exists an integer $c$, such that every planar graph has a $c$-colouring that is nonrepetitive on paths with at most $2 p$ vertices. Note that the bound on $c$ in terms of $p$ here is large. It is open whether there is a polynomial function $f$ such that for every integer $k \geqslant 1$ every planar graph $G$ has a $f(k)$-colouring that is nonrepetitive on paths with most $2 k$ vertices.

Finally, we mention a class of planar graphs that seem difficult to nonrepetitively colour. Let $T$ be a tree rooted at a vertex $r$. Let $V_{i}$ be the set of vertices in $T$ at distance $i$ from $r$. Draw $T$ in the plane with no crossings. Add a cycle on each $V_{i}$ in the cyclic order defined by the drawing to create a planar graph $G_{T}$. It is open whether $\pi\left(G_{T}\right) \leqslant c$ for some constant $c$ independent of $T$. Note that this class of planar graphs includes examples with unbounded degree and unbounded treewidth.

\section{Proof of Theorem 1}

A layering of a graph $G$ is a partition $V_{0}, V_{1}, \ldots, V_{p}$ of $V(G)$ such that for every edge $v w \in E(G)$, if $v \in V_{i}$ and $w \in V_{j}$ then $|i-j| \leqslant 1$. Each set $V_{i}$ is called a layer. The following lemma by Kündgen and Pelsmajer [14] will be useful.

\footnotetext{
${ }^{1}$ One can prove this bound using a naive application of the Lipton-Tarjan planar separator theorem.
} 
Lemma 2 ([14]). For every layering of a graph $G$, there is a (not necessarily proper) 4colouring of $G$ such that for every repetitively coloured path $\left(v_{1}, v_{2}, \ldots, v_{2 t}\right)$, the subpaths $\left(v_{1}, v_{2}, \ldots, v_{t}\right)$ and $\left(v_{t+1}, v_{t+2}, \ldots, v_{2 t}\right)$ have the same layer pattern.

A separation of a graph $G$ is a pair $\left(G_{1}, G_{2}\right)$ of subgraphs of $G$, such that $G=G_{1} \cup G_{2}$. In particular, there is no edge of $G$ between $V\left(G_{1}\right)-V\left(G_{2}\right)$ and $V\left(G_{2}\right)-V\left(G_{1}\right)$.

Lemma 3. Fix $\varepsilon \in(0,1)$ and $c \geqslant 1$. Let $G$ be a graph with $n$ vertices. Fix a layering $V_{0}, V_{1}, \ldots, V_{p}$ of $G$. Assume that, for every set $B \subseteq V(G)$, there is a separation $\left(G_{1}, G_{2}\right)$ of $G$ such that:

- each layer $V_{i}$ contains at most c vertices in $V\left(G_{1}\right) \cap V\left(G_{2}\right) \cap B$, and

- both $V\left(G_{1}\right)-V\left(G_{2}\right)$ and $V\left(G_{2}\right)-V\left(G_{1}\right)$ contain at most $(1-\varepsilon)|B|$ vertices in $B$.

Then $\pi(G) \leqslant 4 c\left(1+\log _{1 /(1-\varepsilon)} n\right)$.

Proof. Run the following recursive algorithm Compute $(V(G), 1)$.

$\operatorname{Compute}(B, d)$

1. If $B=\emptyset$ then exit.

2. Let $\left(G_{1}, G_{2}\right)$ be a separation of $G$ such that each layer $V_{i}$ contains at most $c$ vertices in $V\left(G_{1}\right) \cap V\left(G_{2}\right) \cap B$, and both $V\left(G_{1}\right)-V\left(G_{2}\right)$ and $V\left(G_{2}\right)-V\left(G_{1}\right)$ contain at most $(1-\varepsilon)|B|$ vertices in $B$.

3. Let $\operatorname{depth}(v):=d$ for each vertex $v \in V\left(G_{1}\right) \cap V\left(G_{2}\right) \cap B$.

4. For $i \in[1, p]$, injectively label the vertices in $V_{i} \cap V\left(G_{1}\right) \cap V\left(G_{2}\right) \cap B$ by $1,2, \ldots, c$. Let label $(v)$ be the label assigned to each vertex $v \in V_{i} \cap V\left(G_{1}\right) \cap V\left(G_{2}\right) \cap B$.

5. Compute $\left(\left(V\left(G_{1}\right)-V\left(G_{2}\right)\right) \cap B, d+1\right)$

6. Compute $\left(\left(V\left(G_{2}\right)-V\left(G_{1}\right)\right) \cap B, d+1\right)$

The recursive application of COMPUTE determines a rooted binary tree $T$, where each node of $T$ corresponds to one call to Compute. Associate each vertex whose depth and label is computed in a particular call to COMPUTE with the corresponding node of $T$. (Observe that the depth and label of each vertex is determined exactly once.)

Colour each vertex $v$ by $(\operatorname{col}(v), \operatorname{depth}(v), \operatorname{label}(v))$, where col is the 4-colouring from Lemma 2. Suppose on the contrary that $\left(v_{1}, v_{2}, \ldots, v_{2 t}\right)$ is a repetitively coloured path in $G$. By Lemma $2,\left(v_{1}, v_{2}, \ldots, v_{t}\right)$ and $\left(v_{t+1}, v_{t+2}, \ldots, v_{2 t}\right)$ have the same layer pattern. In addition, $\operatorname{depth}\left(v_{i}\right)=\operatorname{depth}\left(v_{t+i}\right)$ and $\operatorname{label}\left(v_{i}\right)=\operatorname{label}\left(v_{t+i}\right)$ for all $i \in[1, t]$. Let $v_{i}$ and $v_{t+i}$ be vertices in this path with minimum depth. Since $v_{i}$ and $v_{t+i}$ are in the same layer and have the same label, these two vertices were not labelled at the same step of 
the algorithm. Let $x$ and $y$ be the two nodes of $T$ respectively associated with $v_{i}$ and $v_{t+i}$. Let $z$ be the least common ancestor of $x$ and $y$ in $T$. Say node $z$ corresponds to call Compute $(B, d)$. Thus $v_{i}$ and $v_{t+i}$ are in $B$ (since if a vertex $v$ is in $B$ in the call to Compute associated with some node $q$ of $T$, then $v$ is in $B$ in the call to Compute associated with each ancestor of $q$ in $T)$. Let $\left(G_{1}, G_{2}\right)$ be the separation in $\operatorname{Compute}(B, d)$. Since $\operatorname{depth}\left(v_{i}\right)=\operatorname{depth}\left(v_{t+i}\right)>d$, neither $v_{i}$ nor $v_{t+i}$ are in $V\left(G_{1}\right) \cap V\left(G_{2}\right)$. Since $z$ is the least common ancestor of $x$ and $y$, without loss of generality, $v_{i} \in V\left(G_{1}\right)-V\left(G_{2}\right)$ and $v_{t+i} \in V\left(G_{2}\right)-V\left(G_{1}\right)$. Thus some vertex $v_{j}$ in the subpath $\left(v_{i+1}, v_{i+2}, \ldots, v_{t+i-1}\right)$ is in $V\left(G_{1}\right) \cap V\left(G_{2}\right)$. If $v_{j} \in B$ then $\operatorname{depth}\left(v_{j}\right)=d$. If $v_{j} \notin B$ then $\operatorname{depth}\left(v_{j}\right)<d$. In both cases, $\operatorname{depth}\left(v_{j}\right)<\operatorname{depth}\left(v_{i}\right)=\operatorname{depth}\left(v_{t+i}\right)$, which contradicts the choice of $v_{i}$ and $v_{t+i}$. Hence there is no repetitively coloured path in $G$.

Observe that the maximum depth is at most $1+\log _{1 /(1-\varepsilon)} n$. Therefore the number of colours is at most $4 c\left(1+\log _{1 /(1-\varepsilon)} n\right)$.

We now show that a result by Lipton and Tarjan [15] implies the condition in Lemma 3 for planar graphs.

Lemma 4. Let $r$ be a vertex in a connected planar graph $G$. For $i \geqslant 0$, let $V_{i}$ be the set of vertices at distance $i$ from $r$. Then $V_{0}, V_{1}, \ldots, V_{p}$ is a layering of $G$. For every set $B \subseteq V(G)$, there is a separation $\left(G_{1}, G_{2}\right)$ of $G$ such that:

- each layer $V_{i}$ contains at most two vertices in $V\left(G_{1}\right) \cap V\left(G_{2}\right) \cap B$,

- both $V\left(G_{1}\right)-V\left(G_{2}\right)$ and $V\left(G_{2}\right)-V\left(G_{1}\right)$ contain at most $\frac{2}{3}|B|$ vertices in $B$.

Proof. Let $T$ be a breath-first spanning tree in $G$ starting at $r$. Thus, for each vertex $v$, the distance between $v$ and $r$ in $T$ equals the distance between $v$ and $r$ in $G$.

Lipton and Tarjan [15, Lemma 2] proved that for every vertex weighting of $G$ (with non-negative weights totalling at most 1), there is an edge $v w \in E(G)-E(T)$, such that if $C$ is the 'fundamental' cycle consisting of $v w$ and the two paths from $v$ and $w$ back to their least common ancestor in $T$, then the vertices inside $C$ have total weight at most $\frac{2}{3}$, and the vertices outside $C$ have total weight at most $\frac{2}{3}$.

Apply this result with each vertex in $B$ weighted $\frac{1}{|B|}$, and each vertex in $V(G)-B$ weighted 0 . Let $G_{1}$ and $G_{2}$ be the subgraphs of $G$ induced by $C$ and the vertices inside $C$ and outside $C$ respectively. Then $\left(G_{1}, G_{2}\right)$ is a separation. The total weight of $V\left(G_{1}\right)-$ $V\left(G_{2}\right)$ equals the number of vertices in $\left(V\left(G_{1}\right)-V\left(G_{2}\right)\right) \cap B$. Hence $V\left(G_{1}\right)-V\left(G_{2}\right)$, and by symmetry $V\left(G_{2}\right)-V\left(G_{1}\right)$, contains at most $\frac{2}{3}|B|$ vertices in $B$.

Since $T$ is breadth-first, the paths from $v$ and $w$ back to their least common ancestor in $T$ each contain at most one vertex from each layer $V_{i}$. Hence, each layer $V_{i}$ contains at most two vertices in $V\left(G_{1}\right) \cap V\left(G_{2}\right) \cap B$.

Lemmas 3 and 4 together prove Theorem 1 (by adding edges to make $G$ connected).

\section{Acknowledgement}

Thanks to the anonymous referees who pointed us to Lipton and Tarjan's lemma. 


\section{References}

[1] Michael O. Albertson, Glenn G. Chappell, Hal A. Kierstead, André Kündgen, and Radhika Ramamurthi. Coloring with no 2-colored $P_{4}$ 's. Electron. J. Combin., 11 \#R26, 2004. http://www.combinatorics.org/Volume_11/ Abstracts/v11i1r26.html. MR: 2056078.

[2] Noga Alon and Jaroseaw Grytczuk. Breaking the rhythm on graphs. Discrete Math., 308:1375-1380, 2008. doi: 10.1016/j.disc.2007.07.063. MR: 2392054.

[3] Noga Alon, JarosŁaw Grytczuk, Mariusz HaŁuszczak, and Oliver RiORDAN. Nonrepetitive colorings of graphs. Random Structures Algorithms, 21(34):336-346, 2002. doi: 10.1002/rsa.10057. MR: 1945373.

[4] János Barát and PÉter P. VARJú. On square-free vertex colorings of graphs. Studia Sci. Math. Hungar., 44(3):411-422, 2007. doi: 10.1556/SScMath.2007.1029. MR: 2361685.

[5] Boštjan Brešar, JarosŁaw Grytczuk, Sandi Klavžar, StanisŁaw NiWCZYK, AND IZTOK PETERIN. Nonrepetitive colorings of trees. Discrete Math., 307(2):163-172, 2007. doi: 10.1016/j.disc.2006.06.017. MR: 2285186.

[6] Panagiotis Cheilaris, Ernst Specker, and Stathis Zachos. Neochromatica. Comment. Math. Univ. Carolin., 51(3):469-480, 2010. http://www.dml.cz/dmlcz/ 140723. MR: 2741880.

[7] Sebastian Czerwiński and Jaroseaw Grytczuk. Nonrepetitive colorings of graphs. Electron. Notes Discrete Math., 28:453-459, 2007. doi: 10.1016/j.endm.2007.01.063. MR: 2324051.

[8] Vida Dujmović, Gwenaël Joret, Jakub Kozik, and David R. Wood. Nonrepetitive colouring via entropy compression. 2012. arXiv: 1112.5524 .

[9] Jaroseaw Grytczuk. Thue-like sequences and rainbow arithmetic progressions. Electron. J. Combin., 9(1):R44, 2002. http://www.combinatorics.org/Volume_9/ Abstracts/v9i1r44.html. MR: 1946146.

[10] JarosŁaW Grytczuk. Nonrepetitive colorings of graphs - a survey. Int. J. Math. Math. Sci., 74639, 2007. doi: 10.1155/2007/74639. MR: 2272338.

[11] JarosŁaW Grytczuk. Thue type problems for graphs, points, and numbers. Discrete Math., 308(19):4419-4429, 2008. doi: 10.1016/j.disc.2007.08.039. MR: 2433769.

[12] Jaroseaw Grytczuk, Jakub PrzybyŁo, and Xuding Zhu. Nonrepetitive list colourings of paths. Random Structures Algorithms, 38(1-2):162-173, 2011. doi: 10.1002/rsa.20347. MR: 2768888.

[13] Jochen Haranta and Stanislav Jendrol'. Nonrepetitive vertex colorings of graphs. Discrete Math., 312(2):374-380, 2012. doi:10.1016/j.disc.2011.09.027. MR: 2852595.

[14] Andre Kündgen and Michael J. Pelsmajer. Nonrepetitive colorings of graphs of bounded tree-width. Discrete Math., 308(19):4473-4478, 2008. doi: 10.1016/j.disc.2007.08.043. MR: 2433774. 
[15] Richard J. Lipton and Robert E. Tarjan. A separator theorem for planar graphs. SIAM J. Appl. Math., 36(2):177-189, 1979. doi:10.1137/0136016. MR: 0524495.

[16] Dániel Marx and Marcus Schaefer. The complexity of nonrepetitive coloring. Discrete Appl. Math., 157(1):13-18, 2009. doi:10.1016/j.dam.2008.04.015. MR: 2479374.

[17] Jaroslav NešetřIl and Patrice Ossona de Mendez. Tree-depth, subgraph coloring and homomorphism bounds. European J. Combin., 27(6):1022-1041, 2006. doi: 10.1016/j.ejc.2005.01.010. MR:2226435.

[18] Jaroslav Nešetrill, Patrice Ossona de Mendez, and David R. Wood. Characterisations and examples of graph classes with bounded expansion. European J. Combinatorics, 33(3):350-373, 2011. doi: 10.1016/j.ejc.2011.09.008. MR: 2864421.

[19] Axel Thue. Über unendliche Zeichenreihen. Norske Vid. Selsk. Skr. I. Mat. Nat. Kl. Christiania, 7:1-22, 1906.

\section{A Lower Bounds}

Barát and Varjú [4] constructed a planar graph $G$ with $\pi(G) \geqslant 10$. Pascal Ochem [private communication] observed that this lower bound can be improved to 11 by adapting a construction due to Albertson et al. [1] as follows. Barát and Varjú [4] constructed an outerplanar graph $H$ with $\pi(H) \geqslant 7$. Let $G$ be the following planar graph. Start with a path $P=\left(v_{1}, \ldots, v_{22}\right)$. Add two adjacent vertices $x$ and $y$ that both dominate $P$. Let each vertex $v_{i}$ in $P$ be adjacent to every vertex in a copy $H_{i}$ of $H$. Suppose on the contrary that $G$ is nonrepetitively 10-colourable. Without loss of generality, $x$ and $y$ are respectively coloured 1 and 2 . A vertex in $P$ is redundant if its colour is used on some other vertex in $P$. If no two adjacent vertices in $P$ are redundant then at least 11 colours appear exactly once on $P$, which is a contradiction. Thus some pair of consecutive vertices $v_{i}$ and $v_{i+1}$ in $P$ are redundant. Without loss of generality, $v_{i}$ and $v_{i+1}$ are respectively coloured 3 and 4 . If some vertex in $H_{i} \cup H_{i+1}$ is coloured 1 or 2, then since $v_{i}$ and $v_{i+1}$ are redundant, with $x$ or $y$ we have a repetitively coloured path on 4 vertices. Now assume that no vertex in $H_{i} \cup H_{i+1}$ is coloured 1 or 2. If some vertex in $H_{i}$ is coloured 4 and some vertex in $H_{i+1}$ is coloured 3 , then with $v_{i}$ and $v_{i+1}$, we have a repetitively coloured path on 4 vertices. Thus no vertex in $H_{i}$ is coloured 4 or no vertex in $H_{i+1}$ is coloured 3. Without loss of generality, no vertex in $H_{i}$ is coloured 4. Since $v_{i}$ dominates $H_{i}$, no vertex in $H_{i}$ is coloured 3 . We have proved that no vertex in $H_{i}$ is coloured $1,2,3$ or 4 , which is a contradiction, since $\pi\left(H_{i}\right) \geqslant 7$. Therefore $\pi(G) \geqslant 11$. 